\title{
SELF-ASSESSMENT OF THE CONTENT OF THE PUBLIC HEALTH MASTER'S EDUCATIONAL PROGRAM FOR COMPLIANCE WITH THE EUROPEAN PUBLIC HEALTH CORE COMPETENCES PROGRAMME
}

DOI: 10.36740/WLek202103227

\author{
Tetiana S. Gruzieva', Nataliia V. Hrechyshkina' ${ }^{1}$, Olena Ya. Antonyuk' ${ }^{\text {, Vasyl A. Dufynets }}{ }^{2}$, Serhii E. Konovalov' \\ 'BOGOMOLETS NATIONAL MEDICAL UNIVERSITY, KYIV, UKRAINE \\ 2UZHHOROD NATIONAL UNIVERSITY, UZHHOROD, UKRAINE
}

\begin{abstract}
The aim: Determining the compliance of the developed Public Health Educational Program with the principles and basic provisions of the WHO-ASPHER Competency Framework for Public Health Workforce in the European Region.

Materials and methods: The study used bibliographic and information-analytical methods and content analysis. A comparative analysis compliance of the content of the Public Health Master's Educational Program, developed in Bogomolets National Medical University, with the provisions of the WHO-ASPHER Competency Framework for Public Health Workforce in the European Region in terms of providing academic disciplines in the ECTS credits (European credit transfer and accumulation system), has been carried out. Results: Comparative analysis of the components of the University Public Health Master's Educational Program and regulations of the WHO-ASPHER Competency Framework for Public Health Workforce in the European Region has identified a high level of their compliance in all areas of competence formation, including science and practice, health promotion, legislation, policy, ethics, common health and safety in health, leadership and operational mind-set, cooperation and partnership, communication, culture and advocacy, strategic and resource management, professional development, organizational training and adaptability.

The competence-based educational program provides future professionals with theoretical knowledge and practical skills for a clear understanding of public health problems, a reasonable choice of methods for solving them, taking into account modern practices; forming partnerships, effective communication and cooperation on a cross-sectoral basis, developing leadership skills, organizing and providing people-centred public health services.

Conclusions: Self-assessment of the content of Public Health Master's Educational Program for compliance with European educational standards for the formation of the necessary competencies is an important tool for its quality formation and improvement. A comparative analysis of the university's Public Health Master's Educational Program, with the European Competency Framework for Public Health Workforce, has identified a high level of compliance.
\end{abstract}

KEY WORDS: Public Health, workforce training, educational programs, academic disciplines, competencies, functions

Wiad Lek. 2021;74(3 p.II):713-717

\section{INTRODUCTION}

Providing the proper level of health of the population, which requires the improvement of the main determinants of human health and quality of life, the general coverage of health services, is crucial for achieving the social goals set by the Sustainable Development Strategy. [1-2].

The implementation of tasks aimed at improving population health in modern conditions is complicated by a number of problems, including aging, the negative impact of socio-economic and environmental determinants, increasing health inequalities, epidemic of non-infectious and pandemic infectious diseases, uncontrolled migration, etc. [3-7].

Under current conditions, health systems are building their development strategies towards ensuring sustainability, the ability to respond to challenges, the provision of high-quality services focused on the modern needs of consumers, and the rational use of available resources. This requires improving the provision of services, expanding their range, introducing inclusive service models, applying innovative approaches, technical innovations, establishing interagency interaction and the participation of all stakeholders. [8-9].

The current epidemiological landscape of population health causes a high need for public health services as a preventively oriented integrative basis for the prevention of health disorders, its preservation and promotion. Personnel matter have a special place among the priority ones for the successful development of the public health system. They cover various aspects, from determining the needs for human resources and requirements for their quality, including the processes of training, their distribution, use, professional development, etc. [10-12].

The development of human resources in the public health system should correspond to the general approaches to the generation of health resources and specific requirements for knowledge, skills and competencies in this area of activity. In this context, it is especially important to expand the scope of vocational education to lifelong learning and the transition from specialized training to the formation and 
development of a wide range of necessary competencies.

This approach is consistent with the provisions of the Global Strategy on Human Resources for Health: Workforce 2030 and the Regional Committee for Europe Resolution "Towards a sustainable health workforce in the WHO European Region: framework for action". Those documents emphasize the need to develop normative documents to regulate the improvement of public health workforce professional qualifications and to assist in obtaining the necessary skills in specific conditions at the national level. This requires the vocational education system restructuring and additional investment in workforce training [13-14].

In Ukraine, health care reform involves, among other things, prioritizing the development of the public health service and building its human resources. The approval of the new "Public Health" specialty is aimed at the implementation of the educational area. The professional environment has developed and approved educational standards for Public Health Bachelor's and Master's degrees at the state level, which defines the requirements for training specialists. Educational programs and public health workforce training have been developed at a number of universities. The National Classification of Occupations includes the new professions "environmental and health specialist" and "public health specialist". The preparation of the integrated test exam STEP 2 for the "Public Health" specialty and the educational standard for the training of doctors of philosophy is underway [15-16].

Substantiation and development of the Public Health Educational Program by universities in Ukraine was carried out in accordance with state educational standards, requirements of national legislation and existing needs. The recommendations of international organizations have been taken into account, including WHO, Association of Schools of Public Health in the European Region (ASPHER), Agency for Public Health Education Accreditation (APHEA), and experience of many countries in Europe and the world [17-18].

Given the European vector of Ukraine's development and the need to integrate the approaches to solving common cross-border problems in public health sphere, the unity of requirements for the preparation of specialized labour resources, common understanding of ways to preserve and promote health based on existing knowledge, abilities and skills that form the competence basis of specialists.

Although European countries differ significantly in the available capacity and organization of public health services, models of service provision and features of human resources training, the WHO-ASPHER Competency Framework for Public Health Workforce in the European region was developed as a framework document. The proposed document provides ample opportunities for European countries to optimize curricula and programs in the field of public health, to assess existing capacity, to identify needs, to plan investments, to create accreditation systems, to stimulate cooperation, etc. [19].

In view of the above, self-assessment of university vocational educational programs in the field of "public health" allows to compare their provisions with the requirements of the European community, serves as a tool for comparing and identifying deficiencies, shortcomings, and provides opportunities for improvement and modernization.

\section{THE AIM}

The aim was determining the compliance of the developed Public Health Educational Program with the principles and basic provisions of the WHO-ASPHER Competency Framework for Public Health Workforce in the European Region.

\section{MATERIAL AND METHODS}

The study used bibliographic and information-analytical methods and content analysis. The content and structure of the WHO-ASPHER Competency Framework for Public Health Workforce in the European Region, categories and levels of professional qualification have been analysed. The assessment of the main components of the Public Health Master's Educational Program, developed in Bogomolets National Medical University, on their compliance with the provisions of the WHO-ASPHER Competency Framework for Public Health Workforce in the European Region by the following groups: science and practice, health promotion, law, policy, ethics, personal health and health security, leadership and operational mind-set, cooperation and partnership, communication, culture and communication outreach, leadership and resource management, and professional development has been carried out.

\section{RESULTS}

Research of content and structure of the WHO-ASPHER Competency Framework for Public Health Workforce in the European Region allows forming a new approach to the assessment of Public Health Master's Educational Programs in terms of the formation of future professionals with a wide range of competencies required to perform professional duties in European countries. It is suggested to consider the competency-based public health workforce in three categories, namely: contents and context, attitudes and interactions, performance and achievements. These categories of competencies include 10 sections, which cover 84 individual competencies. The category "content and context" includes science, knowledge and theory related to public health practice; the category of "relationship and interaction" includes the competence of communication, cooperation, networking to achieve leadership goals and partnerships; the category "results of work and achievements" includes competences for decision-making and activities to gain health. Thus, professional qualifications of public health workforce provide three levels, namely: competency, specialist, expert.

The set of competencies in the section "science and practice" involves the availability of knowledge and skills in a wide range of sciences, including the epidemiology 
of infectious and non-infectious diseases; demographics; biostatistics; qualitative and quantitative research methods; analysis and assessment; evidence-based medicine; health care organizations; population health and health inequalities.

Health competencies are based on knowledge and skills in education; literacy in population, group and individual health; knowledge of the rights and opportunities of citizens; health service needs assessments; application of screening and secondary prevention; evaluations of interventions and health promotion programs results.

The section of legislation, policy and ethics competencies covers knowledge and application of international and European legislation and regulations in the public health sphere; strategies and programs; development of policies and plans; their assessment; setting priorities; decision-making in compliance with ethical norms.

Under the heading of "Common Health Principle and Health Safety", the competence is based on knowledge of human health; health care; international health; global risks and threats; cross-border healthcare; environmental and climate change hygiene; occupational hygiene; food safety.

The category of "relationship and interaction" includes competencies that provide leadership qualities and operational mind-set, cooperation and partnership, communication, culture, advocacy work.

Competency basis in the category "results of work and achievements" is formed by knowledge and skills in strategic leadership and resource management, professional development and reflective practice in compliance with ethical standards, organizational issues and the ability to adapt.

Public Health Master's Educational Program, prepared by Bogomolets National Medical University, in the amount of 120 ECTS credits, includes 34 compulsory and 10 elective courses. Their study is aimed at ensuring the formation of the necessary competencies for prospective Public Health Masters' successful practical activities.

A comparative analysis of the university curriculum with the European Competency Framework for Public Health Workforce has been conducted in each of the 10 areas.

It was found that the educational content of the educational program in "Science and Practice" provides 13 disciplines, including Epidemiology of Infectious Diseases, Epidemiology of Non-infectious Diseases, Demography, Biostatistics, Research Methodology, Modern Methods of Data Analysis, Monitoring and Evaluation of Interventions, Obtaining, Visualization and Aggregation of Public Health Data, Global Burden of Disease, Public Health, Organization and Management in Health Care, Health Care Systems, and Health Inequalities. 20 ECTS credits have been allocated for their mastering. The components of the university curriculum in this area fully comply with the WHO-ASPHER Competency Framework for Public Health Workforce in the European Region.

To acquire competencies in the field of "Health Promotion" there are 6 academic disciplines in the university curriculum, namely: Healthy Lifestyle Promotion; Communication and Mass Media; Forms of Medical Attention, Public Health
Prevention, Monitoring and Evaluation of Interventions, Assessment and Forecasting of Health Care Needs. The volume of learning is 10 ECTS credits. These disciplines give you an opportunity to master the knowledge and skills provided by the WHO-ASPHER Competency Framework for Public Health Workforce in the European Region, in terms of training and support through public participation; health literacy issues at the community, organization and individual levels; screening and secondary prevention; assessments of interventions and health support programs; health care needs assessments.

There is educational content in "Legislation, Strategy, and Ethics" area in the following disciplines of the university curriculum: Law in the Health Care System; Public Health Policy; Ethics in Public Health. 8 ECTS credits are allocated for mastering the material and developing competencies in international, European and national legislation, developing strategies and using strategic approaches, developing programs, evaluation and implementation policies, applying ethical frameworks, implementing ethical practices and decision-making, planning and setting priorities.

Considerable attention in the university's Public Health Master's Educational Program is focused on "Common Health Principle and Health Safety". The list of academic disciplines allows covering all the issues provided by the WHO-ASPHER Competence Framework for Public Health Workforce in the European Region in this area, including human health; health protection; occupational health; international health; global risks and threats; pandemics; environmental health; safe food; climate change and health. To do this, the learning process involves the acquisition of 13 disciplines, such as Social and Environmental Determinants of Health, Public Health, Global Burden of Disease; Pedagogy of Health; Psychology of Health; Sociology of Health; Sexology; Physical Education and Health; Sanitary and Epidemiological Supervision; Infection Control; Occupational Safety and Health; Occupational Risk Methodology; International Health Problems, Health Care Emergencies; Environmental Protection. The volume of academic disciplines is 30 ECTS credits.

Inclusion in the educational program of educational components of competences in the group "Leadership qualities and operational mind-set" is achieved through the following disciplines: Public Health Policy (intersectoral strategies); Healthy Lifestyle Promotion; Public Health Management and Leadership; Public Health in Practice; Human Resources Management; Strategic Management in Health Care. The study of these subjects gives you an opportunity to form the competencies regulated by the WHO-ASPHER Competency Framework for Public Health Workforce in the European Region, namely: Visions, Missions and Strategies; Individual Work in the Target Group; Leadership in Change and Innovation; Understanding and using theories of Complex Systems in Practice; Organizational Training and Development; Human Development; Emotional Development. 10 ECTS credits have been allocated for this in the educational program. 
In accordance with "Cooperation and Partnership" area of the compared WHO-ASPHER Competency Framework, the university curriculum provides for the formation of competencies for effective cooperation; building relationships and partnerships; building interdisciplinary and intersectoral networks, relationships with stakeholders and their management within the study of "Health Care Policy" discipline.

The formation of competencies in "Communication, Culture and Advocacy" area is due to the inclusion in the educational process of the following studying disciplines: Communication and Media; Health Care Media and Systems; Acquisition, Visualization and Compiling Data in Public Health. They allow you to acquire knowledge and skills in effective written and verbal communication, including communication with the media; presentations; communication outreach, etc.

The content of the educational program in "Strategic leadership and resource management" area is provided by 8 academic disciplines, including Human Resources Management; Quality Management in Health Care System; Managerial Accounting; Health Care System Funding; Financial Analysis and Assessment of the Financial Condition of the Medical Organization; Fundamentals of Logistics; Economics; Pharmacoeconomics. 14 ECTS credits have been allocated for their mastering. The components of the university curriculum are fully compliant with the WHO-ASPHER Competency Framework for Public Health Workforce in the European Region on human resource management; quality assurance; administration and resource management; financial planning; technical expertise and logistics; economic evaluation and analysis.

According to "Professional development and reflective practice in compliance with ethics" area, the teaching of Human Resources and Public Health Ethics disciplines is provided for in the university curriculum, laying the foundations for continuous professional development, lifelong learning and ethical professional behaviour.

In order to acquire competencies in "Literacy in Organizational Matters and the Ability to Adapt" area 1 academic discipline, including Entering into Contracts and Financing of Medical Services; Fundamentals of Accounting; Medical Insurance; Acquisition, Visualization and Compiling Data in Public Health; Marketing of Medical Services; Capital Market; Modern Methods of Data Analysis; Health Information Tools and Systems; Telemedicine and E-health are allocated in the university curriculum. The volume of learning is 22 ECTS credits. These disciplines allow you to master the knowledge and skills provided by the WHO-ASPHER Competency Framework for Public Health Workforce in the European Region, on the use of technology; data management; entrepreneurship; creativity, analysis and synthesis; digital health and social media; understanding of services and operations in public health.

\section{DISCUSSION}

A comparative analysis of the university's Public Health Master's Educational Program with the European Compe- tency Framework for Public Health Workforce have found a high level of compliance. Virtually all competencies in the 10 areas of core competencies provided by the WHOASPHER Competency Framework for Public Health Workforce in the European Region are provided by the educational context of the university curriculum while studying a wide range of disciplines. At the same time, attention is paid to the formation of competencies in such aspects of the future activities of Public Health Masters as a deep understanding of public health matters and theories on which the practice is based; establishing the necessary connections, effective cooperation and implementation of leadership qualities; substantiation, development and decision making for gaining in health with a focus on people's needs.

It goes without saying that health inequalities in the structure and organization of public health services in different countries will determine the features of public health workforce training. However, the strategic directions of educational training should ensure the formation of a basic, mandatory list of competencies, without which successful activities in public health are impossible. This is the approach offered by WHO-ASPHER in the WHOASPHER Competency Framework for Public Health Workforce in the European Region. It presents a clear normative interpretation of the needs in the competence-based of the public health workforce for the implementation of the tasks assigned to them.

In view of the above, the periodic assessment of Public Health Master's Educational Programs for their compliance with the general requirements for acquiring the necessary competencies should become a routine practice of improving the formation of public health human resources.

\section{CONCLUSIONS}

Self-assessment of Public Health Workforce Educational Programs for their compliance with WHO-ASPHER Competency Framework for Public Health Workforce in the European Region is an important tool for their quality formation and promotion. The use of this tool will allow assessing the overall educational program, identifying its strengths and possible shortcomings, deficiencies with the necessary competencies of prospective Public Health Bachelors or Masters.

A comparative analysis of the Public Health Workforce Educational Program developed by Bogomolets National Medical University, with the European Competency Framework for Public Health Workforce has found a high level of compliance. The list of academic disciplines available in the educational program creates preconditions for the formation of almost all the necessary competencies in 10 main areas. Competence-based educational program will give an opportunity to acquire theoretical knowledge and master practical skills providing the prospective professionals with clear understanding of public health problems, reasonably choosing methods to solve them with respect to current practices; creating partnerships, commu- 
nicating and collaborating effectively on a cross-sectoral basis, demonstrating leadership skills, and organizing and delivering people-centred public health services.

\section{REFERENCES}

1. Transforming our world: the 2030 Agenda for Sustainable Development. United Nations. 2015. https://sustainabledevelopment.un.org/ post2015/transformingourworld/publication\#.

2. Tracking universal health coverage: First global monitoring report. Geneva: World Health Organization. 2015.

3. European health report 2018 «More than numbers - evidence for all». Copenhagen:WHO EURO; 2018.

4. Evidence and resources to act on health inequities, social determinants and meet the SDGs. Copenhagen:WHO EURO; 2019.

5. Health inequityand the effects of COVID-19. Copenhagen:WHO EUR0;2020.

6. Protecting health in Europe from climate change: 2017 update. Copenhagen:WHO EURO; 2017.

7. Gruzieva T.S., Diachuk M.D., Inshakova H.V. et al. Modern demographic trends in Ukraine as a realization of preventional strategies. Wiadomości Lekarskie. 2019;72:2033-2039.

8. European Action Plan for Strengthening Public Health Capacities and Services. Copenhagen: WHO Regional Office for Europe; 2015.

9. Implementing a Health 2020 vision: governance for health in the $21 \mathrm{st}$ century. Making it happen. Copenhagen: WHO Regional Office for Europe; 2013.

10. Strengthening public health services across the European Region - a summary of background documents for the European Action Plan. Copenhagen: WHO Regional Office for Europe; 2012.

11. Second expert meeting of The Coalition of Partners to Strengthen Public Health Capacities and Services in the European Region: how do we collaborate powerfully and accelerate targeted action? Copenhagen: WHO Regional Office for Europe; 2017.

12. Rechel B., McKee M. Facets of Public Health in Europe. Copenhagen: WHO Regional Office for Europe; 2014.

13. Global strategy on human resources for health: Workforce 2030. DRAFT 1.0 submitted to the Executive Board (138th Session). Geneva: World Health Organization; 2016.

14. Towards a sustainable health workforce in the WHO European Region: framework for action. Copenhagen: WHO Regional Office for Europe. 2017. https://www.euro.who.int/_data/assets/pdf_ file/0011/343946/67wd10e_HRH_Framework_170677.pdf?ua=1.

15. Gruzieva T.S., Pelo I., Smiyanov V.A. et al. Conceptual assumptions to create a system for preparation of healthcare human resources in Ukraine. Wiadomości Lekarskie. 2016;69:719-725.
16. Gruzeva T., Kurylo T. Educational Requirements and Practice of Public Health Professionals Training in the Republic of Poland as a Basis for Formation of Educational Programs in Ukraine. In: Health problems in Ukraine and Poland. Lviv: Editorial House of the Lviv Regional Charity Fund "Medicine and Law"; 2017. p. 46-52.

17. ASPHER's European List of Core Competences for the Public Health Professional. Scandinavian Journal of Public Health. 2018;46(23):1-52. doi:10.1177/1403494818797072.

18. Curriculum validation. Handbook \& application. Version 3.6.-2020. Agency for Public Health Education Accreditation; 2020.

19. WHO-ASPHER Competency Framework for the Public Health Workforce in the European Region. Copenhagen:WHO Regional Office for Europe; 2020.

The article was performed in framework of research "Medical and social substantiation of the optimization of the healthcare organization in the context of the public healthcare system development", (2020-2022, № of state registration 0120U100807).

\section{ORCID and contributionship:}

Tetiana S. Gruzieva: 0000-0001-9254-7561 A,B,C,D,E,F

Nataliia V. Hrechyshkina: 0000-0002-9499-4362 ${ }^{A, B, D, E}$

Olena Ya. Antonyuk: 0000-0002-3411-196X ${ }^{B}$

Vasyl A. Dufynets: 0000-0001-8754-6177 ${ }^{E}$

Serhii E. Konovalov: 0000-0001-7462-3970 ${ }^{B}$

\section{Conflict of interest:}

The Authors declare no conflict of interest.

\section{CORRESPONDING AUTHOR Tetiana S. Gruzieva}

Bogomolets National Medical University

13 Taras Shevchenko Boulevard, 01601 Kyiv, Ukraine

e-mail:gruzieva@ukr.net

Received: 13.12 .2020

Accepted: 05.03.2021

A - Work concept and design, B - Data collection and analysis, C - Responsibility for statistical analysis, D-Writing the article, E-Critical review, $\mathbf{F}$ - Final approval of the article 